\title{
A new three-dimensional chaotic system with a hidden attractor, circuit design and application in wireless mobile robot
}

\author{
SUNDARAPANDIAN VAIDYANATHAN, ACENG SAMBAS, MUSTAFA MAMAT and MADA SANJAYA WS
}

This research work proposes a new three-dimensional chaotic system with a hidden attractor. The proposed chaotic system consists of only two quadratic nonlinearities and the system possesses no critical points. The phase portraits and basic qualitative properties of the new chaotic system such as Lyapunov exponents and Lyapunov dimension have been described in detail. Finally, we give some engineering applications of the new chaotic system like circuit simulation and control of wireless mobile robot.

Key words: chaos, chaotic systems, hidden attractors, circuit simulation.

\section{Introduction}

Chaos theory describes nonlinear dynamical systems that are very sensitive to initial conditions. Since the experimental discovery of a chaotic system by Lorenz [16], chaos theory has found applications in several areas in science and engineering $[1,2,3,4,5$, $6,7,8,9,10,11,12]$.

Recently, chaotic attractors have been classified as self-excited or hidden attractors $[13,14]$. An attractor is said to be self-excited provided its basin of attraction overlaps with neighborhood of a critical point. Chaotic attractors, which are not self-excited, are known as hidden attractors.

Some well-known examples of self-excited chaotic attractors are Chen system [15], Lorenz system [16], Sundarapandian systems [17, 18], Pehlivan system [19], Akgul system [20], etc.

Sundarapandian Vaidyanathan, the corresponding author is with Research and Development Centre, Vel Tech University, Avadi, Chennai- 600062, Tamil Nadu, India, e-mail: sundarvtu@ gmail.com. Aceng Sambas is with Faculty of Informatics and Computing, Universiti Sultan Zainal Abidin, Malaysia, and Department of Mechanical Engineering, Universitas Muhammadiyah Tasikmalaya, Indonesia, e-mail: acengs@umtas.ac.id. Mustafa Mamat is with Faculty of Informatics and Computing, Universiti Sultan Zainal Abidin, Malaysia, e-mail: must@unisza.edu.my. Mada Sanjaya WS is with Department of Physics, UIN Sunan Gunung Jati Bandung, Indonesia, e-mail: madasws@gmail.com.

Received 10.07.2017. Revised 2.11.2017. 
Some well-known examples of hidden chaotic attractors are chaotic systems with no critical points [21, 22, 23] or with only one stable critical point [24].

This research article proposes a new three-dimensional chaotic system with a hidden attractor. It is interesting that the new chaotic system has no critical points. The proposed chaotic system consists of only two quadratic nonlinearities and these nonlinearities appear in the second and third differential equations of the system.

In Section 2, we describe the dynamics, phase plots and also the dynamical properties of the new chaotic system with hidden attractor in detail. The high value of Lyapunov dimension establishes the high complexity of the proposed chaotic system with hidden attractor. In Sections 3 and 4, we give some engineering applications of the new chaotic system with hidden attractor such as circuit simulation and control of wireless mobile robot. Section 5 draws the main conclusions of this research article.

\section{A new chaotic system with a hidden attractor}

This work proposes a new autonomous three-dimensional system with two quadratic nonlinearities

$$
\left\{\begin{array}{l}
\dot{x}_{1}=x_{2} \\
\dot{x}_{2}=-x_{1}-x_{2}-a x_{2} x_{3} \\
\dot{x}_{3}=b x_{2}^{2}-c x_{1}-d
\end{array}\right.
$$

where $x_{2} x_{3}$ and $x_{1}^{2}$ are the two quadratic nonlinearities in the system dynamics.

We show that the system (1) is chaotic when we take

$$
a=0.1, b=0.05, c=0.1, d=1
$$

Also, for the values of the parameters given in Eq. (2), the Lyapunov exponents of the system (1) are determined by applying Wolf's algorithm [25] as

$$
L_{1}=0.02207, L_{2}=0, L_{3}=-0.02432
$$

Also, the Lyapunov dimension of the new chaotic system (1) is calculated as

$$
D_{L}=2+\frac{L_{1}+L_{2}}{\left|L_{3}\right|}=2.9075,
$$

which shows the high complexity of the system.

Thus, the 3-D chaotic system (1) has a strange attractor.

For MATLAB plots of the chaotic system (1), we take the initial state as $X(0)=$ $[0.2,0.2,0.2]^{T} \in \mathbf{R}^{3}$. We keep the parameter values as in the chaotic case (2).

Figure 1 portrays the three-dimensional view of the new chaotic system (1) in $\mathbf{R}^{3}$.

Figures 2-4 portray the two-dimensional projections of the new three-dimensional chaotic system $(1)$ in $\left(x_{1}, x_{2}\right),\left(x_{2}, x_{3}\right)$ and $\left(x_{1}, x_{3}\right)$ planes, respectively. 


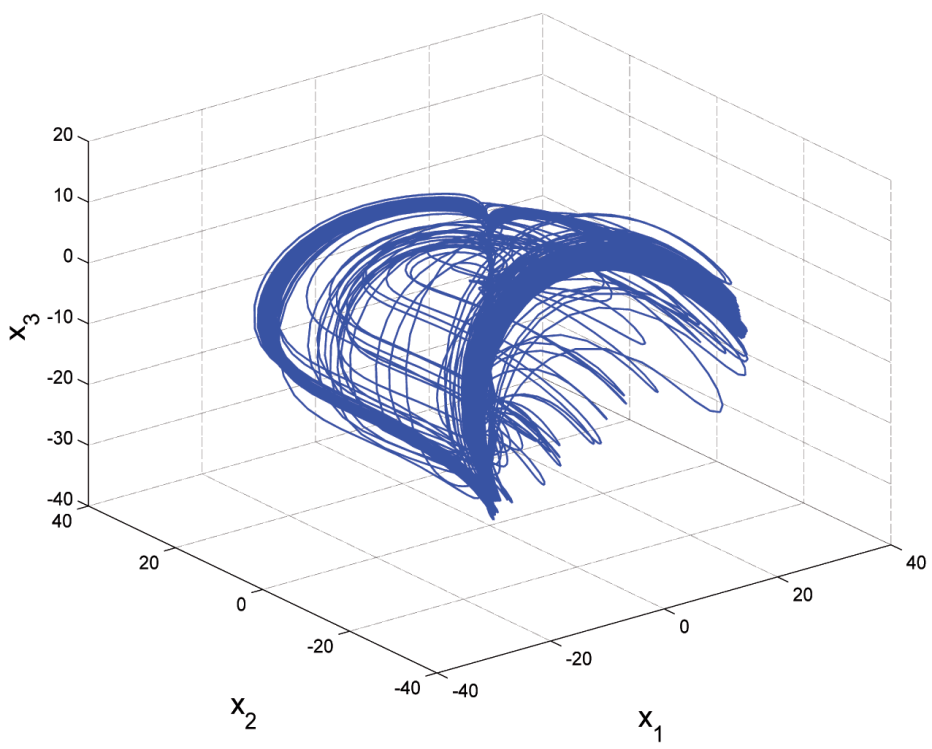

Figure 1: A three-dimensional view of the new chaotic system (1) in $\mathbf{R}^{3}$ for $a=0.1, b=$ $0.05, c=0.1, d=1$

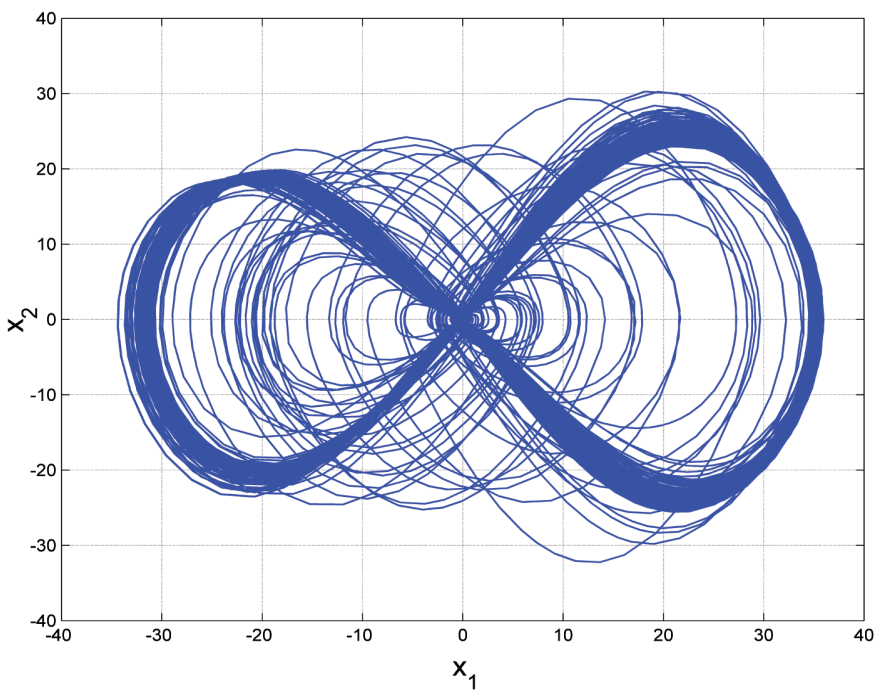

Figure 2: A two-dimensional view of the new chaotic system (1) in $\left(x_{1}, x_{2}\right)$-plane for $a=0.1, b=0.05, c=0.1, d=1$ 


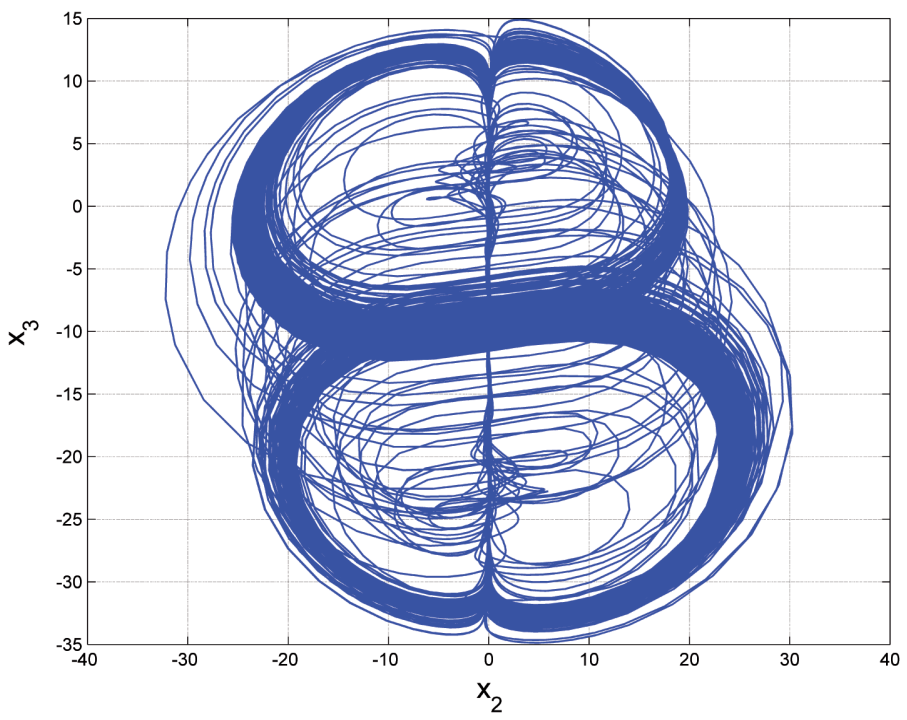

Figure 3: A two-dimensional view of the new chaotic system (1) in $\left(x_{2}, x_{3}\right)$-plane for $a=0.1, b=0.05, c=0.1, d=1$

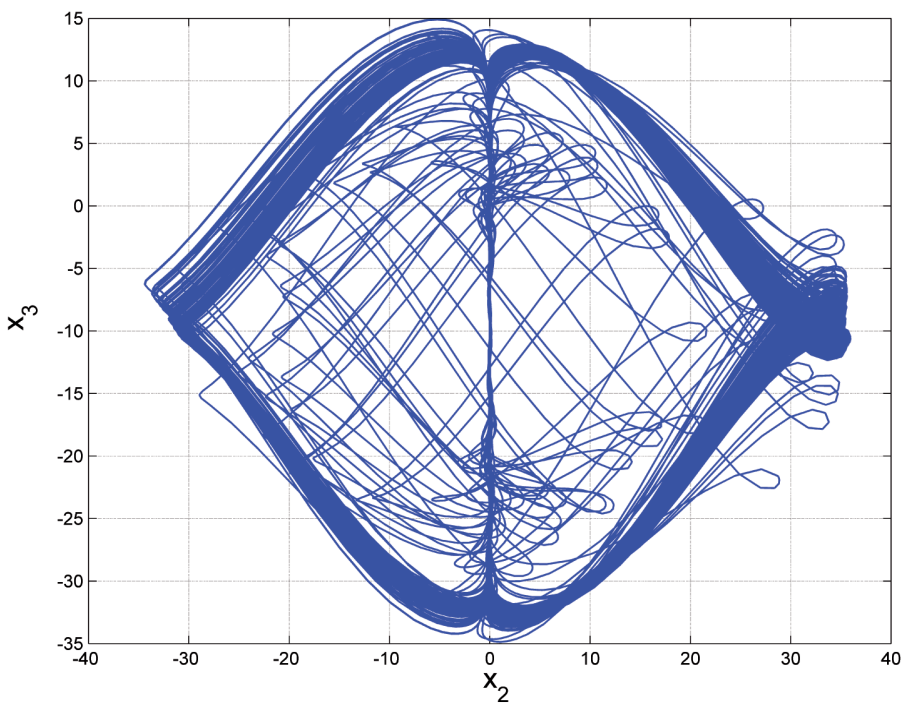

Figure 4: A two-dimensional view of the new chaotic system (1) in $\left(x_{1}, x_{3}\right)$-plane for $a=0.1, b=0.05, c=0.1, d=1$ 
The critical points of the new three-dimensional chaotic system (1) are determined by solving the system of equations

$$
\begin{aligned}
x_{2} & =0 \\
-x_{1}-x_{2}-a x_{2} x_{3} & =0 \\
b x_{2}^{2}-c x_{1}-d & =0
\end{aligned}
$$

From (5a), we get $x_{2}=0$.

Substituting $x_{2}=0$ in (5b), we get $x_{1}=0$.

Substituting $x_{1}=0$ and $x_{2}=0$ in (5c), we get $d=0$, which is a contradiction in the chaotic case (2).

Thus, the new chaotic system (1) does not have any critical point. Hence, the new chaotic system (1) admits a hidden attractor [13, 14].

\section{Circuit realization of the new chaotic system}

In this section, an electronic circuit is designed to implement the new chaotic system (1).

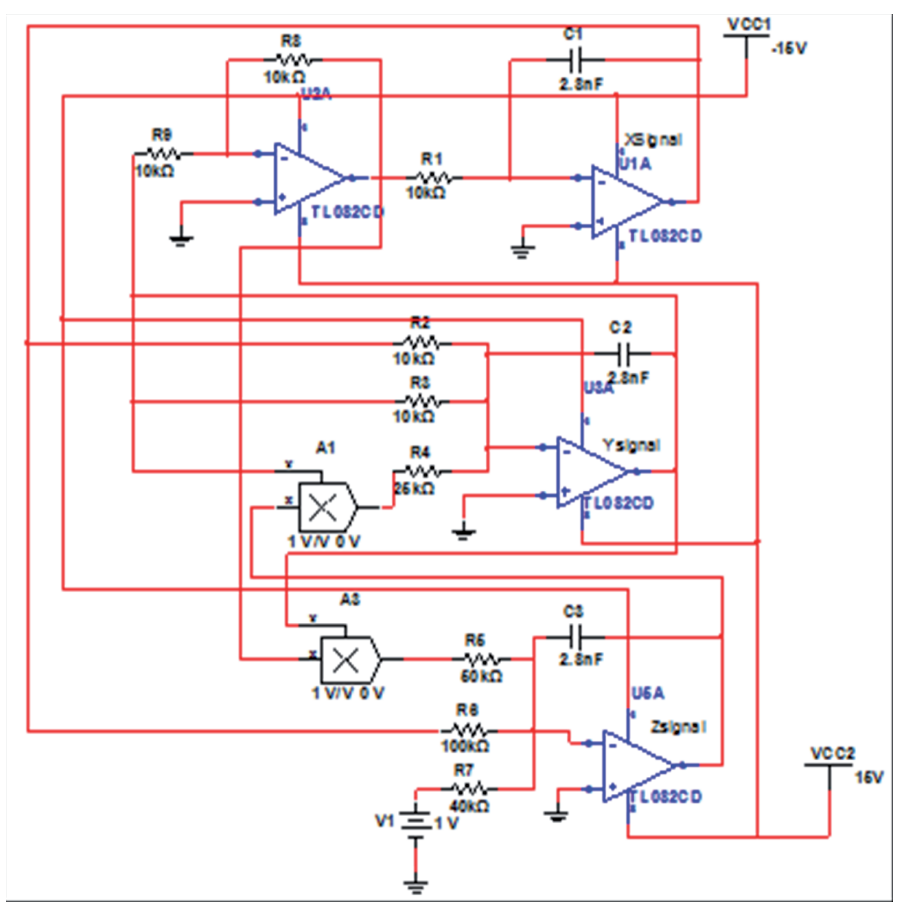

Figure 5: Schematic of the proposed new chaotic system by using MultiSIM 10 
The circuit in Figure 5 has been designed following a general approach based on operational amplifiers $[26,27,28,29]$. The variable $x, y$ and $z$ of the new chaotic system (1) are the voltage across the capacitor $C_{1}, C_{2}$ and $C_{3}$. By applying Kirchhoff's circuit laws, the corresponding circuit equation can be described as:

$$
\begin{aligned}
\frac{d V_{C 1}}{d t} & =\frac{1}{C_{1} R_{1}} V_{C 2} \\
\frac{d V_{C 2}}{d t} & =-\frac{1}{C_{2} R_{2}} V_{C 1}-\frac{1}{C_{2} R_{3}} V_{C 2}-\frac{1}{10 C_{2} R_{4}} V_{C 2} V_{C 3} \\
\frac{d V_{C 3}}{d t} & =\frac{1}{10 C_{3} R_{5}} V_{C 2}^{2}-\frac{1}{C_{3} R_{6}} V_{C 1}-\frac{1}{C_{3} R_{7}} V_{1}
\end{aligned}
$$

The electronic components are chosen as: $R_{1}=R_{2}=R_{3}=R_{8}=R_{9}=10 \mathrm{~K} \Omega, R_{4}=$ $25 \mathrm{~K} \Omega, R_{5}=50 \mathrm{~K} \Omega, R_{6}=100 \mathrm{~K} \Omega, R_{7}=40 \mathrm{~K} \Omega, C_{1}=C_{2}=C_{3}=C_{4}=2.8 \mathrm{nF}$ and $V_{1}=1 V_{D C}$.

The designed circuit is implemented in the electronic simulation package MultiSIM and the phase portraits of the chaotic attractor in the circuit are presented in Figures 6-8. Obtained results show that the designed circuit emulates well the theoretical model (1).

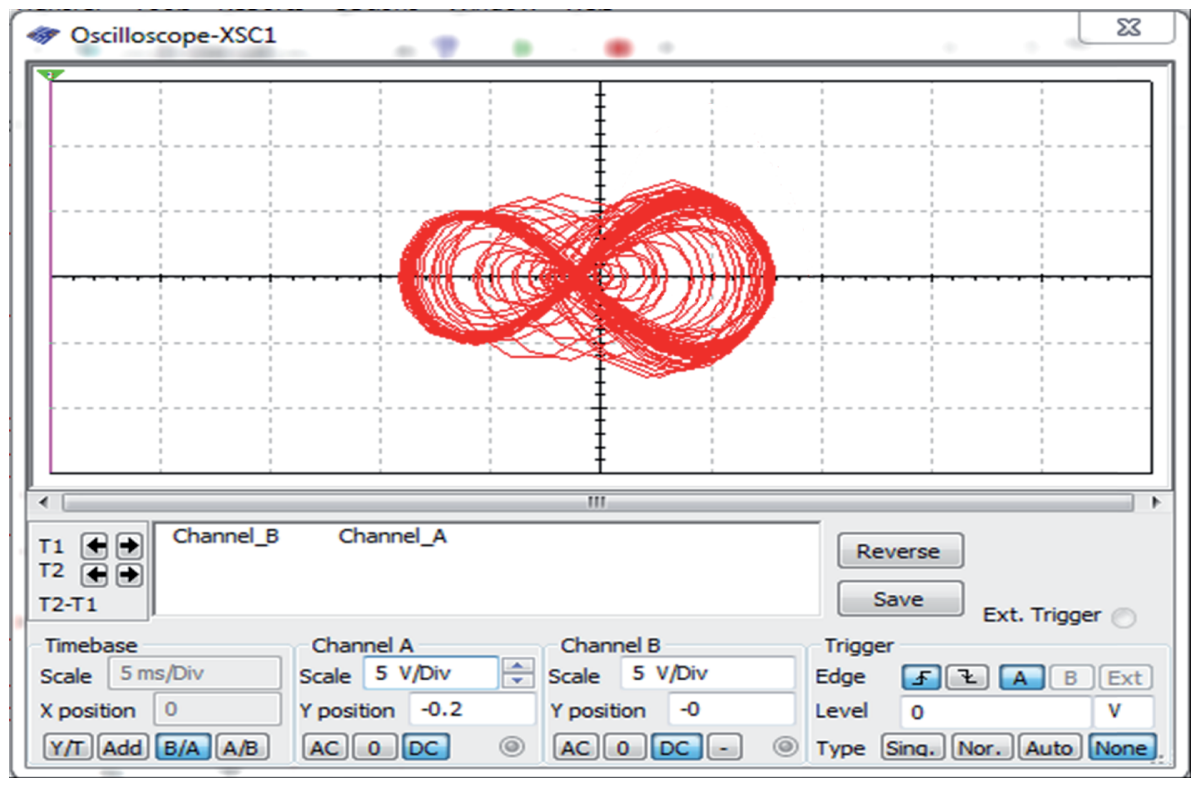

Figure 6: Two-dimensional projection of the new chaotic system in $\left(x_{1}, x_{2}\right)$-plane by using MultiSIM 10 


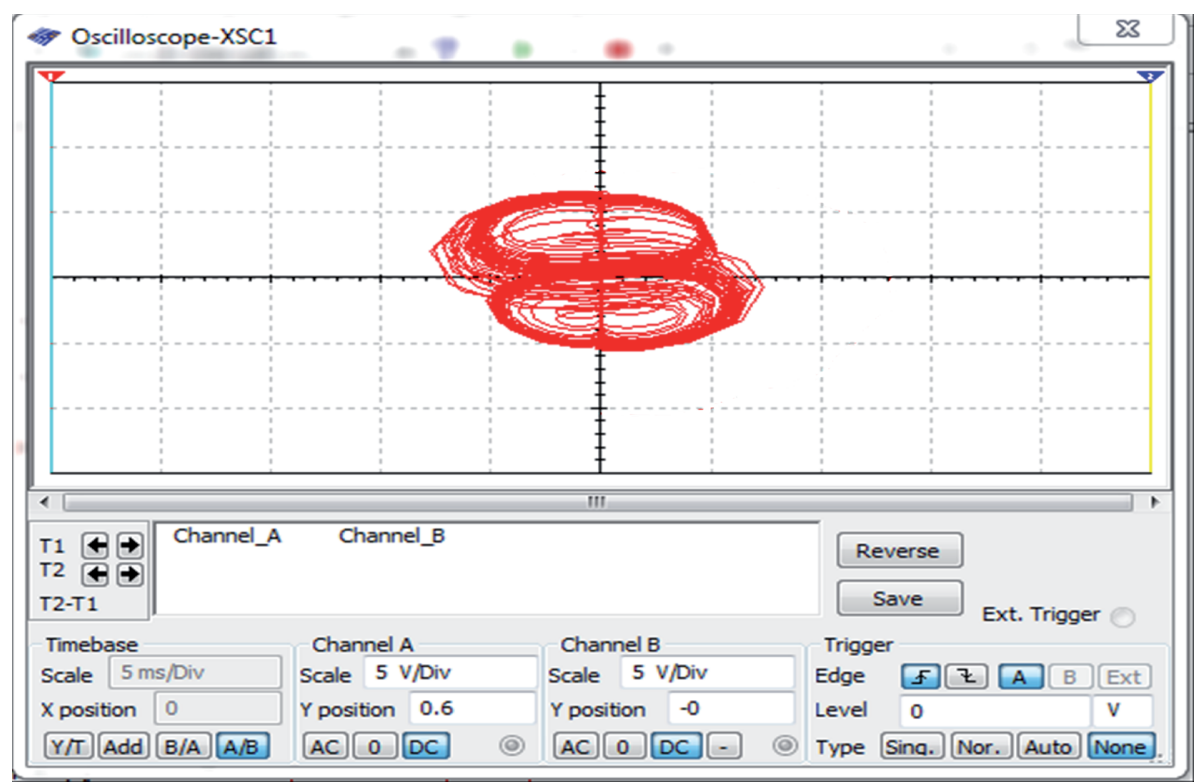

Figure 7: Two-dimensional projection of the new chaotic system in $\left(x_{1}, x_{3}\right)$-plane by using MultiSIM 10

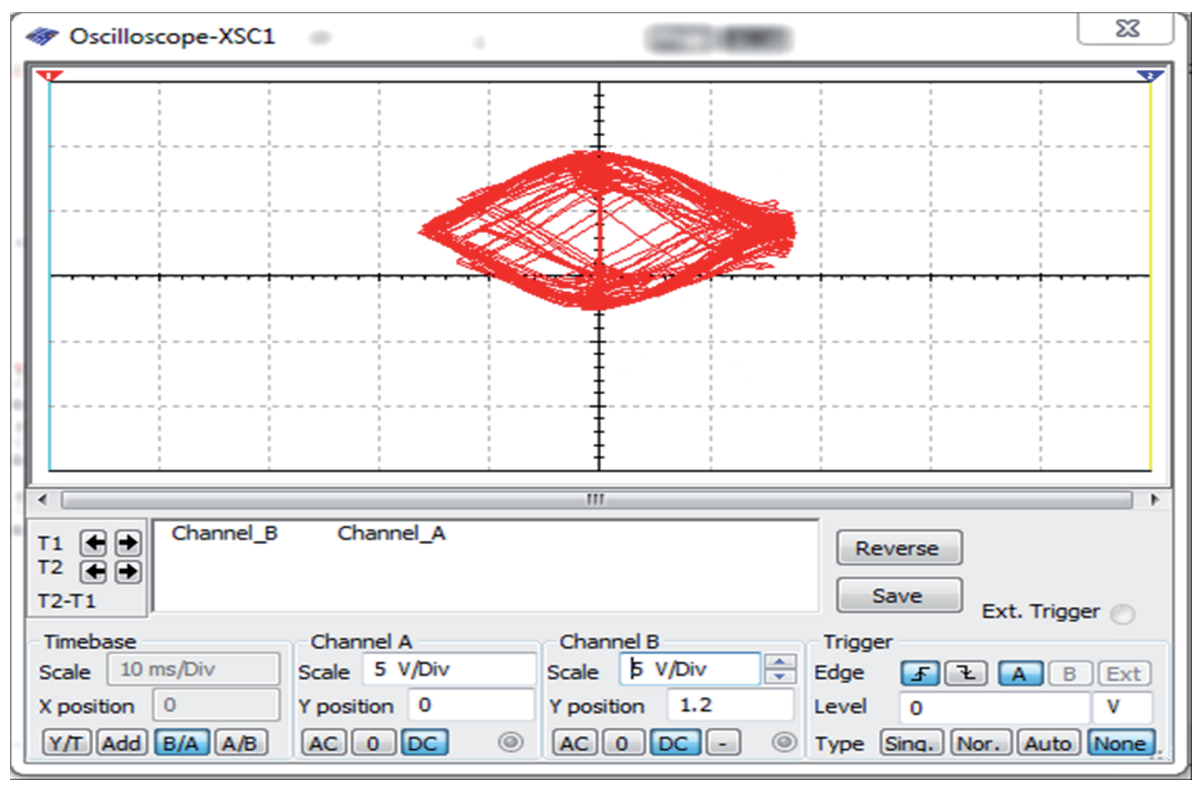

Figure 8: Two-dimensional projection of the new chaotic system in $\left(x_{2}, x_{3}\right)$-plane by using MultiSIM 10 


\section{Application in Wireless Mobile Robot}

In the last decade, the interaction between chaos theory and mobile robots has been studied intensively, such as chaotic behaviour on a fire fighting robot [30], floor-cleaning operation robot [31], patrol mobile robot [32], motion control of robots using a chaotic truly random bits generator [33] and chaotic mobile robot using fuzzy logic [34].

In previous research [35], we have managed to apply mobile robot navigation system with chaos control. The result [35] exhibited the chaotic trajectory with the higher coverage rate of a specific terrain. A weakness of our previous research is delivery of data from the computer to the Arduino still using USB. Therefore, we propose a mobile robot navigation system with data transmission using wireless communications such as Bluetooth device.

The state equations of the mobile robot navigation are written as follows [36]:

$$
\begin{aligned}
\dot{X} & =v(t) \cos \theta(t) \\
\dot{Y} & =v(t) \sin \theta(t) \\
\dot{\theta} & =w(t)
\end{aligned}
$$

where $v(t)=\frac{1}{2}\left(v_{r}(t)+v_{l}(t)\right)$ is the linear velocity of the robot, $v_{r}(t)$ is linear velocity of right wheel, $v_{l}(t)$ is linear velocity of left wheel, $w(t)=\frac{v_{r}(t)-v_{l}(t)}{L}$ is angular velocity and $L$ is distance between the two wheels.

The mobile robot navigation equations were solved numerically by using the fourth order Runge-Kutta algorithm. In the equations of linear velocity and angular velocity of the robot, linear velocity of right wheel $\left(v_{r}(t)\right)$ and linear velocity of left wheel $\left(v_{l}(t)\right)$ are replaced by chaotic signals $x_{1}(t)$ and $x_{2}(t)$, respectively. Then the mobile robot equations become:

$$
\begin{aligned}
v(t) & =\frac{1}{2}\left(x_{1}(t)+x_{2}(t)\right) \\
w(t) & =\frac{x_{1}(t)-x_{2}(t)}{L}
\end{aligned}
$$

By combining Eqs. (1) and (7), the following dynamics is obtained.

$$
\left\{\begin{aligned}
\dot{x}_{1} & =x_{2} \\
\dot{x}_{2} & =-x_{1}-x_{2}-a x_{2} x_{3} \\
\dot{x}_{3} & =b x_{2}^{2}-c x_{1}-d \\
\dot{X} & =v(t) \cos \theta(t) \\
\dot{Y} & =v(t) \sin \theta(t) \\
\dot{\theta} & =w(t)
\end{aligned}\right.
$$

The system (9) describes the mobile robot navigation based on the new chaotic system. The chaotic mobile robot trajectory shown in Figure 9 can be obtained by solving the system dynamics (9) by taking the parameter values and initial conditions as: 


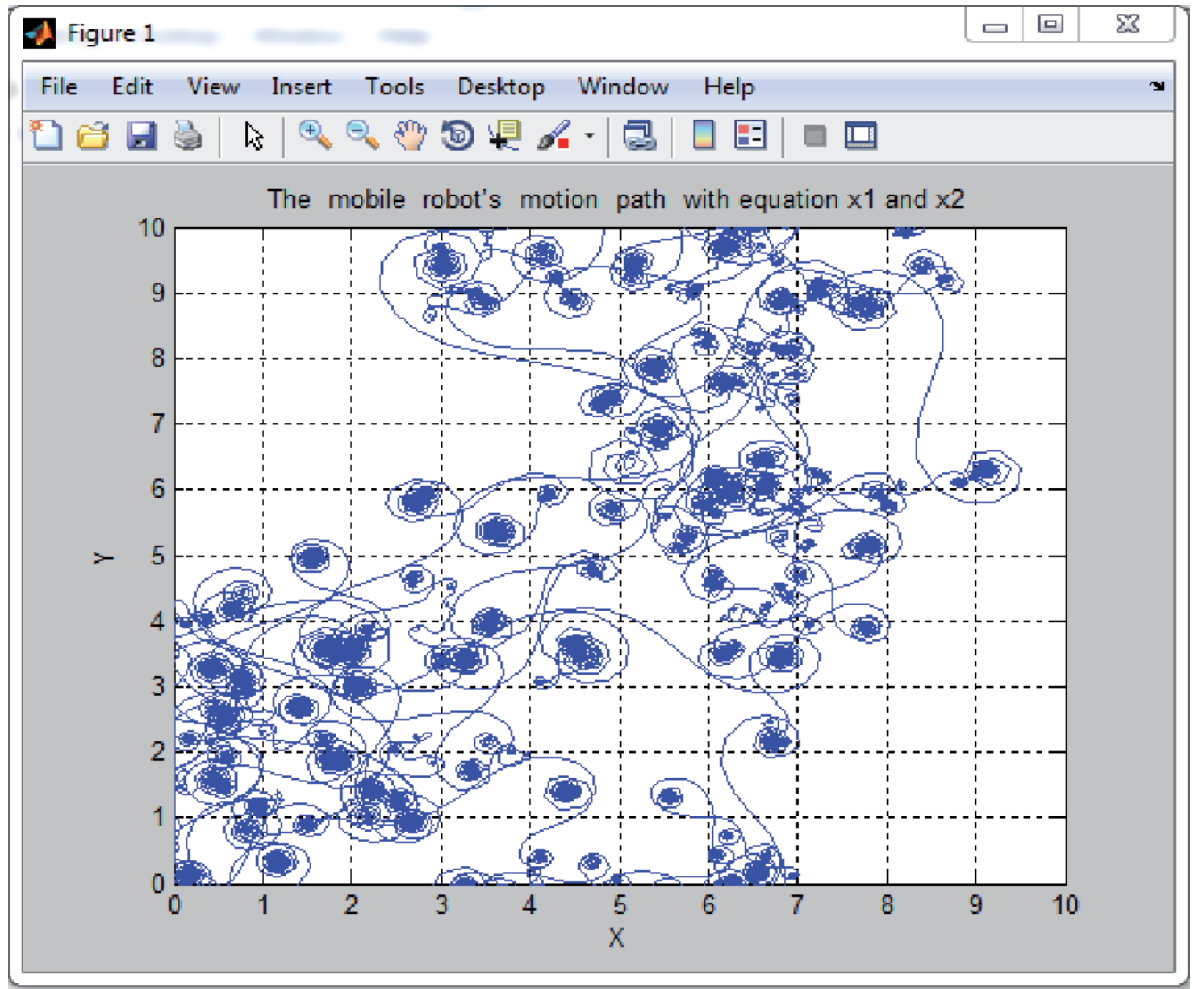

Figure 9: Trajectory of the wireless mobile robot navigation of new chaotic system using MATLAB

$$
\begin{gathered}
a=0.1, b=0.05, c=0.1, d=1, L=0.08 \\
\left(x_{1}, x_{2}, x_{3}\right)=(0.2,0,2,0.2), \quad\left(X_{0}, Y_{0}, \theta_{0}\right)=(0,0,0) .
\end{gathered}
$$

Control wireless mobile robot using the new chaotic system with equation $x_{1}$ and $x_{2}$ that is sent in bluetooth on arduino shows significantly higher value of coverage rate, where the $67 \%$ of the terrain shows to be covered by the robot. The mobile robot's workplace is supposed to be a square terrain with dimensions $M=10 \mathrm{~m} \times 10 \mathrm{~m}=100 \mathrm{~m}^{2}$ in normalized unit cells showed greater than previous research [35].

We develop a wireless mobile robot controlled chaos signal based on bluetooth. Chaos signal from the computer via bluetooth is sent to the Arduino and then Arduino sends a command in the form of PWM to drive the linear velocity of right wheel and linear velocity of left wheel. In this case, bluetooth is used instead of USB as a messenger from computer to robot. Figure 10 shows the circuit schematic of the wireless mobile robot. Figure 11 shows the experimental set-up of the autonomous wireless mobile robot based bluetooth device. The new 3D chaotic system has better performance which is obvious in Figure 12, where the terrain shows to be covered by the wireless 


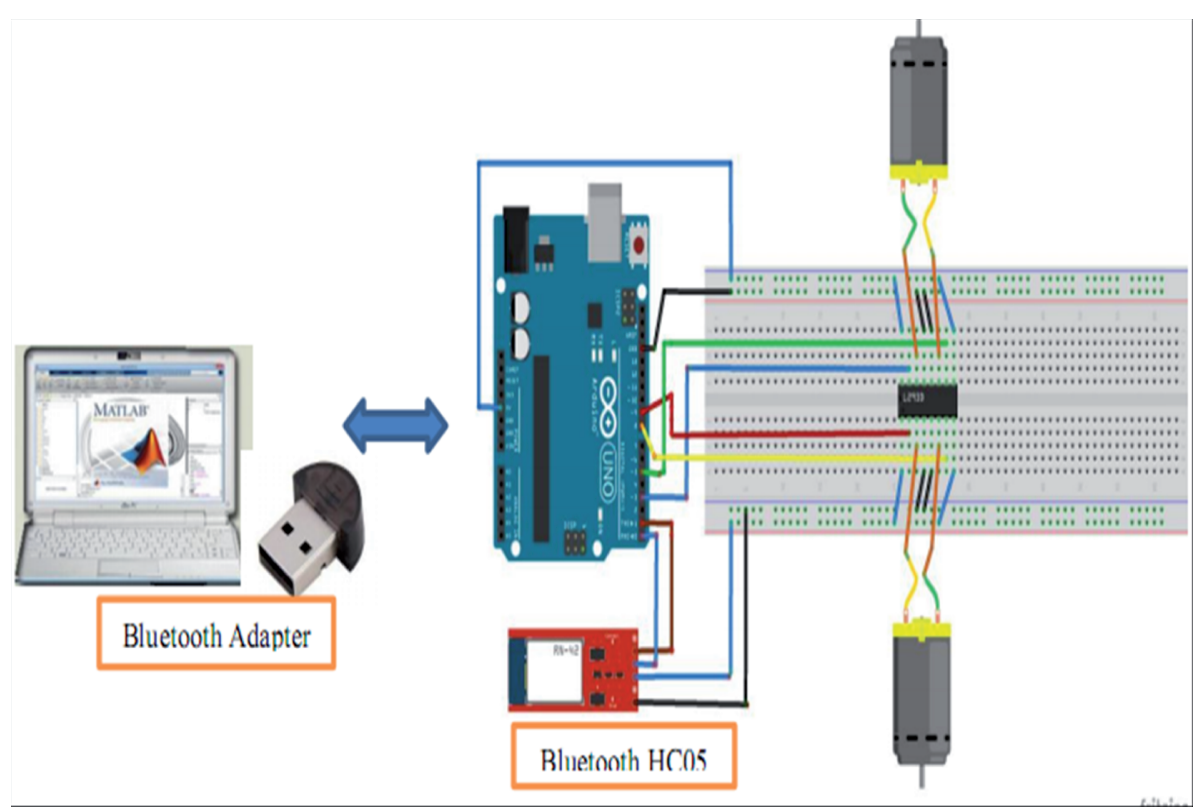

Figure 10: Schematic of the wireless mobile robot

mobile robot. There was little difference in the trajectory of robot simulations with experiments. This is because the data sent from the chaos signal on the robot to activate the motor is very small. Meanwhile, in experiments to activate the motor required input PWM (pulse-width modulation) value in the interval 60-225. Another difference is caused due to the type of motor used.

\section{Conclusion}

A new three-dimensional chaotic system with hidden attractor is constructed and analyzed. The new chaotic system does not have any critical point. The Lyapunov dimension of the new chaotic system is obtained as $D_{L}=2.9075$, which shows the high complexity of the system. Finally, an autonomous wireless mobile robot based bluetooth device by using a chaotic motion controller was presented. The experimental results show an unpredictable trajectory and trajectory with the higher coverage rate of a specific terrain. As future research, we plan to apply chaos signals on robot vision. 


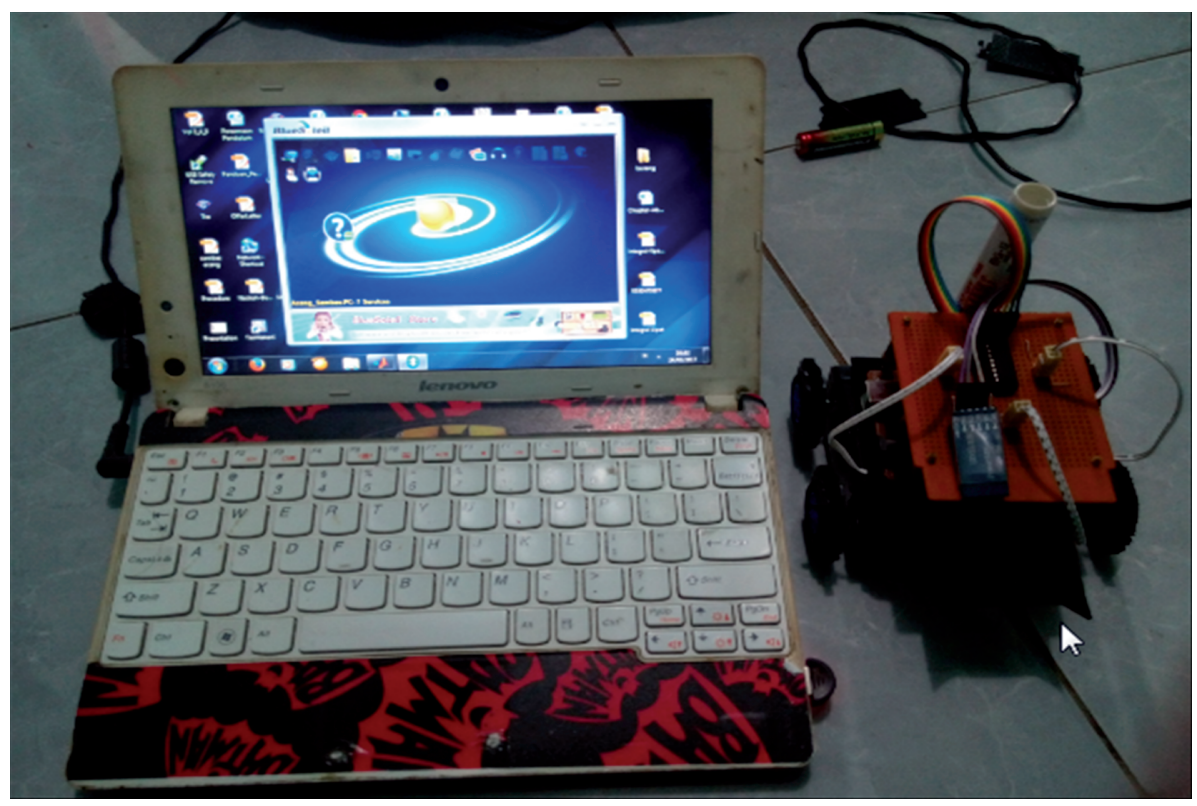

Figure 11: The chaotic autonomous model of wireless mobile robot

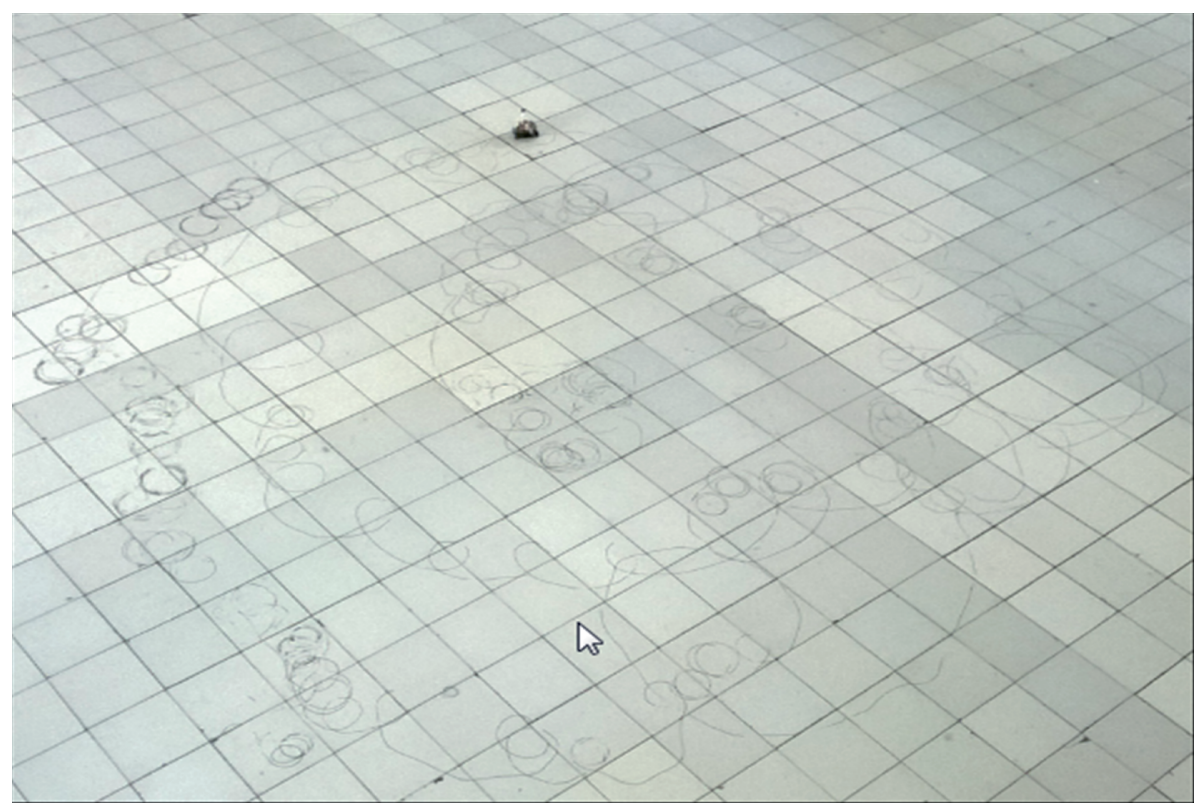

Figure 12: The experimental results of the wireless mobile robot motion based bluetooth device 


\section{References}

[1] S. VAIDYANATHAN and C. VOLOS: Advances and Applications in Chaotic Systems. Springer, Berlin, Germany, 2016.

[2] A.T. AZAR and S. VAIDYANATHAN: Advances in Chaos Theory and Intelligent Control. Springer, Berlin, Germany, 2016.

[3] S. VAIDYANATHAN and C. VOLOS: Advances and Applications in Nonlinear Control Systems. Springer, Berlin, Germany, 2016.

[4] A.T. AZAR, S. VAIDYANATHAN and A. OUANNAS: Fractional Order Control and Synchronization of Chaotic Systems. Springer, Berlin, Germany, 2017.

[5] S. VAIDYANATHAN and C. VOLOS: Advances in Memristors, Memristive Devices and Systems. Springer, Berlin, Germany, 2017.

[6] S. VAIDYANATHAN and C.H. LIEN: Applications of Sliding Mode Control in Science and Engineering. Springer, Berlin, Germany, 2017.

[7] S. RASAPPAN and S. VAIDYANATHAN: Global chaos synchronization of WINDMI and Coullet chaotic systems by backstepping control. Far East Journal of Mathematical Sciences, 67 (2), (2012), 265-287.

[8] S. VAIDYANATHAN, C.K. VOLOS and V.T. PHAM: Global chaos control of a novel nine-term chaotic system via sliding mode control. Studies in Computational Intelligence, 576 (2015), 571-590.

[9] S. VAIDYANATHAN: Adaptive synchronization of novel 3-D chemical chaotic reactor systems. International Journal of ChemTech Research, 8 (7), (2015), 159171.

[10] S. VAIDYANATHAN, C.K. VOLOS, K. RAJAGOPAL, I.M. KYPRIANIDIS and I.N. STOUBOULOS: Adaptive backstepping controller design for the antisynchronization of identical WINDMI chaotic systems with unknown parameters and its SPICE implementation. Journal of Engineering Science and Technology Review, 8 (2), (2015), 74-82.

[11] S. VAIDYANATHAN: Output regulation of the forced Van der Pol chaotic oscillator via adaptive control method. International Journal of PharmTech Research, 8 (6), (2015), 106-116.

[12] S. VAIDYANATHAN: A new 3-D jerk chaotic system with two cubic nonlinearities and its adaptive backstepping control. Archives of Control Sciences, 27 (3), (2017), 409-439. 
[13] D. DUDKOWSKI, S. JAFARI, T. KAPITANIAK, N.V. KUZNETSOV, G.A. LEONOV and A. PRASAD: Hidden attractors in dynamical systems. Physics Reports, 637 (2016), 1-50.

[14] D. DUDKOWSKI, A. PRASAD and T. KAPITANIAK: Perpetual points and hidden attractors in dynamical systems. Physics Letters A, 379 (2015), 2591-2596.

[15] G. CHEN and T. UETA: Yet another chaotic attractor. International Journal of Bifurcation and Chaos, 9 (1999), 1465-1466.

[16] E.N. LORENZ: Deterministic nonperiodic flow. Journal of the Atmospheric Sciences, 20 (1963), 130-141.

[17] V. SUNDARAPANDIAN and I. PEHLIVAN: Analysis, control, synchronization, and circuit design of a novel chaotic system. Mathematical and Computer Modelling, 55 (2012), 1904-1915.

[18] S. VAIDYANATHAN,: Analysis, control, and synchronization of a 3-D novel jerk chaotic system with two quadratic nonlinearities. Kyungpook Mathematical Journal, 55 (3), (2015), 563-585.

[19] I. PEHLIVAN, I.M. MOROZ and S. VAIDYANATHAN: Analysis, synchronization and circuit design of a novel butterfly attractor. Journal of Sound and Vibration, $\mathbf{3 3 3}$ (2014), 5077-5096.

[20] A. AKGUL, I. MOROZ, I. PEHLIVAN and S. VAIDYANATHAN: A new fourscroll chaotic attractor and its engineering applications. Optik, 127 (2016), 54915499 .

[21] V.T. PHAM, C.K. VOLOS and S. JAFARI: Hidden attractors in a chaotic system with an exponential nonlinear term. European Physical Journal: Special Topics, 224 (2015), 1507-1517.

[22] S. VAIDYANATHAN, V.T. PHAM and C.K. VOLOS: A 5-D hyperchaotic Rikitake dynamo system with hidden attractors. European Physical Journal: Special Topics, 224 (2015), 1575-1592.

[23] S. VAIDYANATHAN and C. VOLOS: Analysis and adaptive control of a novel 3-D conservative no-equilibrium chaotic system. Archives of Control Sciences, 25 (3), (2015), 333-353.

[24] X. WANG and G. CHEN: A chaotic system with only one stable equilibrium. Communications in Nonlinear Science and Numerical Simulation, 17 (2012), 1264 1272.

[25] A. WOLF, J.B. SWIFT, H.L. SWINNEY and J.A. VASTANO: Determining Lyapunov exponents from a time series. Physica D, 16 (1985), 285-317. 
[26] X.F. LI, K.E. CHLOUVERAKIS and D.L. XU, Nonlinear dynamics and circuit realization of a new chaotic flow: a variant of Lorenz, Chen and Lu. Nonlinear Analysis: Real World Applications, 10 (2015), 2357-2368.

[27] V.T. PHAM, S. JAFARI, C. VOLOS, A. GIAKOUMIS, S. VAIDYANATHAN and T. KAPITANIAK: A chaotic system with equilibria located on the rounded square loop and its circuit implementation. IEEE Transactions on Circuits and Systems II: Express Briefs, 63 (9), (2016), 878-882.

[28] C. VOLOS, J.O. MAAITA, S. VAIDYANATHAN, V.T. PHAM, I. STOUBOULOS and I. KYPRIANIDIS: A novel four-dimensional hyperchaotic four-wing system with a saddle-focus equilibrium. IEEE Transactions on Circuits and Systems II: Express Briefs, 64 (3), (2017), 339-343.

[29] S. VAIDYANATHAN, A. SAMBAS, M. MAMAT and M. SANJAYA WS: Analysis, synchronisation and circuit implementation of a novel jerk chaotic system and its application for voice encryption. International Journal of Modelling, Identification and Control, 28 (2), (2017), 153-166.

[30] M.J.M. TAVERA, M.S. DUTRA, E.Y.V. DIAZ and O. LENGERKE: Implementation of chaotic behaviour on a fire fighting robot. In Proc. of the 20th Int. Congress of Mechanical Engineering, Gramado, Brazil, November (2015).

[31] J. PALACIN, J.A. SALSE, I. VALGANON and X. CLUA: Building a mobile robot for a floor cleaning operation in domestic environments. IEEE Transactions on Instrumentation and Measurement, 53 (2004), 1418-1424.

[32] L.S. MARTINS FILHO and E.E.N. MACAU: Patrol mobile robots and chaotic trajectories. Mathematical Problems in Engineering, 2007 (2007), Article ID 61543.

[33] C.K. VOLOS, I.M. KYPRIANIDIS and I.N. STOUBOULOS: Motion control of robots using a chaotic truly random bits generator. Journal Engineering Science and Technology Review, 5 (2012), 6-11.

[34] J. NI and S.X. YANG: A fuzzy-logic based chaos GA for cooperative foraging of multi-robots in unknown environments. International Joournal of Robotics and Automation, 27 (2012), 15-30.

[35] A. SAMBAS, S. VAIDYANATHAN, M. MAMAT, M. SANJAYA and D.S. RAHAYU: A 3D novel jerk chaotic system and its application in secure communication system and mobile robot navigation. Studies in Computational Intelligence 636 (2016), 283-310.

[36] V.M. PERI: Fuzzy Logic Controller for an Autonomous Mobile Robot, PhD Thesis, Jawaharlal Nehru Technological University, Hyderabad, India, (2005). 